\title{
Cavernous Gastric Hemangioma as an Unusual Cause of Upper Gastrointestinal Bleeding in a Young Woman
}

\author{
Andrew Sun ${ }^{a}$ Jeffrey Sun ${ }^{b}$ Cheuk-Kay Sun ${ }^{c, d, e, f}$ \\ aEmory College of Arts and Sciences, Emory University, Atlanta, GA, USA; 'bSchool

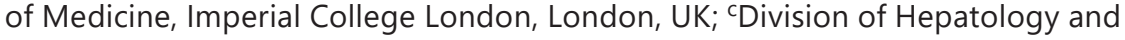 \\ Gastroenterology, Department of Internal Medicine, Shin Kong Wu Ho-Su Memorial \\ Hospital, Taipei, Taiwan; ${ }^{\mathrm{d}} \mathrm{Graduate}$ Institute of Business Administration, Fu Jen Catholic \\ University, Taipei, Taiwan; eSchool of Medicine, Fu Jen Catholic University, Taipei, Taiwan; \\ fSchool of Medicine, Taipei Medical University, Taipei, Taiwan
}

\author{
Keywords \\ Cavernous gastric hemangioma - Gastric cancer - Gastrointestinal bleeding · \\ Esophagogastroduodenoscopy · Gastrectomy · Case report
}

\begin{abstract}
Gastric hemangiomas $(\mathrm{GHs})$ are extremely rare vascular lesions of mesodermal origin that may occur in isolation or in conjunction with underlying congenital pathology. Due to the scarcity of these tumors, there is no standardized diagnostic method; however, many have found the combination of endoscopic investigation and radiographic imaging to be most effective, with the presence of phleboliths on computerized tomography as being pathognomonic for GHs. Surgical treatment for symptomatic lesions is curative with no reports of recurrence. We describe a 21-year-old woman who presented with epigastric pain and one episode of $250 \mathrm{~mL}$ hematemesis earlier that morning. Under the impression of an upper gastrointestinal bleed due to peptic ulcer disease, esophagogastroduodenoscopy was performed which revealed a $5-\mathrm{cm}$ blood clot-like mass similar in appearance to that of a II-b peptic ulcer, but the presence of a bridging fold led to the suspicion of a possible submucosal tumor. Dynamic computerized tomography scan showed similar findings, and the patient was referred for surgical intervention. Laparoscopic distal gastrectomy was performed with the final diagnosis of cavernous GH made via histological evaluation. The patient was discharged 9 days later with no complications. This case puts emphasis on the importance of considering cavernous $\mathrm{GH}$ as a potential cause of severe upper $\mathrm{Gl}$ bleeding especially in those with atypical demographic profile and history.
\end{abstract}




\section{Introduction}

Gastric hemangiomas (GHs) are defined as benign vascular lesions of gastric origin with associated concurrent endothelial cell proliferation. These lesions are extremely rare, representing $1.7 \%$ of all benign tumors of gastric origin and accounting for $0.05 \%$ of all gastrointestinal neoplasms [1]. GHs are commonly classified histologically into capillary, cavernous, and mixed-type $[2,3]$. While some hemangiomas may be found incidentally, symptomatic patients typically present with epigastralgia and dyspepsia with coexistent symptoms of upper gastrointestinal (GI) bleeding such as anemia, hematemesis, and melena. Although these benign lesions are at low risk of malignant transformation, as many as $90 \%$ of patients with cavernous GH may experience sudden onset overt bleeding and sometimes massive lifethreatening hemorrhage [2].

Diagnostic modality is largely dependent on the size of the lesion, with initial investigation being endoscopy followed by computerized tomography (CT) or magnetic resonance imaging (MRI) [4]. Surgery is the definitive treatment for GHs, with confirmation of diagnosis via post-resection histological evaluation. Whilst case records showed that GHs can occur at any age, most of the current literature is dominated by cases in pediatric and elderly patients [5]. We have identified an absence of literature regarding cases of GHs in early adulthood. This article reports a case of isolated gastric cavernous hemangioma in an underreported age group with unusual endoscopic appearance, successfully treated with surgery.

\section{Case Report}

A 21-year-old woman with no relevant past medical history presented to the emergency department in the morning with complaints of persistent epigastric pain since the night before. The patient also reported of one episode of $250 \mathrm{~mL}$ coffee-ground emesis containing blood clots but denied any previous history of hematemesis or melena. On clinical examination, the patient's blood pressure was 101/63 mm Hg with a heart rate of $112 \mathrm{bpm}$ and a respiratory rate of 18 breaths per min. Her abdomen was soft with normoactive bowel sounds and mild epigastric tenderness with no muscle guarding, no palpable mass, and no organomegaly.

The patient was resuscitated with 500 mL lactated Ringer's solution, followed by transfusion with packed red blood cells and fresh frozen plasma alongside tranexamic acid, proton pump inhibitors, and anti-emetics. Initial laboratory tests revealed a hemoglobin concentration of $8.7 \mathrm{~g} / \mathrm{dL}$ (normal: 11.0-16.0 g/dL), hematocrit 35.4\% (normal: 35.0-48.0\%), mean cell volume $85.7 \mathrm{fL}$ (normal 81.0-98.0 fL), platelet $267 \times 10^{3} / \mathrm{uL}$ (normal 140-450 $\times 10^{3} / \mathrm{uL}$ ), INR 1.13 (normal 0.8-1.20), and APTT $28.4 \mathrm{~s}$ (normal 26-40 s). No other abnormalities were detected in other blood parameters. The patient's age and the combination of epigastralgia with anemia and hematemesis led to the possible diagnosis of upper gastrointestinal bleeding due to peptic ulcer disease (PUD) for which urgent esophagogastroduodenoscopy (EGD) was performed. EGD (GIF-Q290; Olympus, Tokyo, Japan) showed large quantities of fresh blood in the stomach and a 5-cm mass similar in appearance to a bleeding ulcer with an adherent clot on the posterior wall of the gastric antrum (shown in Fig. 1a). However, a bleeding submucosal tumor was suspected upon identification of a bridging fold at the base of the lesion, indicating the need for surgical intervention. Dynamic abdominal CT scan showed an active bleeding ulcerative mass with focally obscured borders, measuring $5.2 \mathrm{~cm}$ at the gastric antrum posterior wall (shown in Fig. 1b, c). With the tentative diagnosis of gastric submucosal tumor, laparoscopic distal gastrectomy with Billroth-I anastomosis was performed. A $15.5 \times 7 \times 1.5 \mathrm{~cm}$ specimen from resection containing the vascular lesion, with size consistent

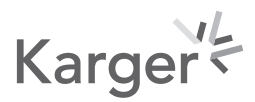



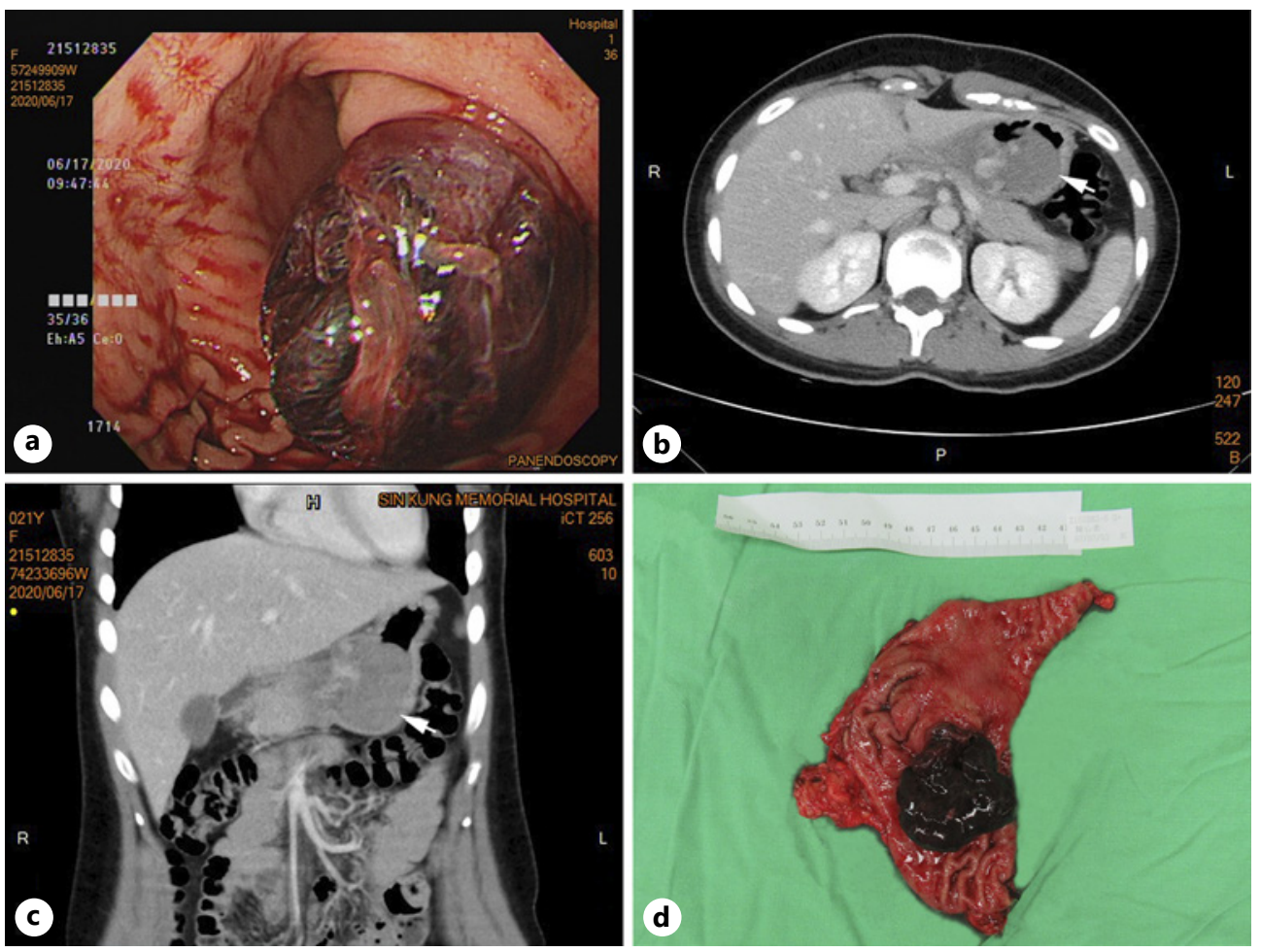

Fig. 1. a Endoscopic view demonstrating a mass on the posterior wall of the gastric antrum with a bridging fold at the base of the lesion. $\mathbf{b}$ Axial view of dynamic abdominal CT scan showing a bleeding ulcerative mass with focally obscured borders at posterior wall of gastric antrum (arrow). c Coronal view of dynamic abdominal CT scan showing the same lesion (arrow). d Resection specimen containing the vascular lesion at the lesser curvature.

with CT and EGD findings, from the stomach along the lesser curvature was subject to pathological analysis that revealed a hemorrhagic protruding polypoid tumor measuring $5 \times 4 \times 2.5$ $\mathrm{cm}$ consistent with the appearance of an ulcerated cavernous hemangioma with hemorrhage (shown in Fig. 1d). Microscopic examination showed a polypoid tumor displaying surface ulceration, adjacent to normal gastric tissue, along with thin-walled, dilated, and congested vessels within the tumor itself (shown in Fig. 2a, b). Further immunohistochemical studies identified tumor cells showing positive staining for CD34 (shown in Fig. 3a) and negative staining for CD117 (shown in Fig. 3b), DOG-1 (shown in Fig. 3c), and beta-catenin (shown in Fig. 3d), thus ruling out GI stromal tumor and confirming the diagnosis of GH. The patient experienced no postoperative complications and was discharged 9 days later.

\section{Discussion}

GHs are congenital hamartomatous tumors of mesodermal origin that can be found throughout the alimentary tract with possible associations with concurrent systemic or cutaneous hemangiomas [2]. These benign tumors are lesions formed by a collection of dilated vessels as a result of endothelial cell proliferation with concomitant pericytic hyperplasia or abnormal pericyte division [6]. GHs are extremely rare vascular lesions first described by Lammers in 1893 [5] and can occur either as a single isolated lesion, or as multiple lesions when associated with underlying congenital abnormalities such as Klippel-Trenaunay-Weber 
Fig. 2. a Hematoxylin and eosin staining of the gastric specimen $(\times 40)$ demonstrating a polypoid tumor with surface ulceration (arrow). b A higher magnification $(\times 200)$ of the same specimen under H\&E staining revealing thin-walled, dilated, and congested vessels (arrows).
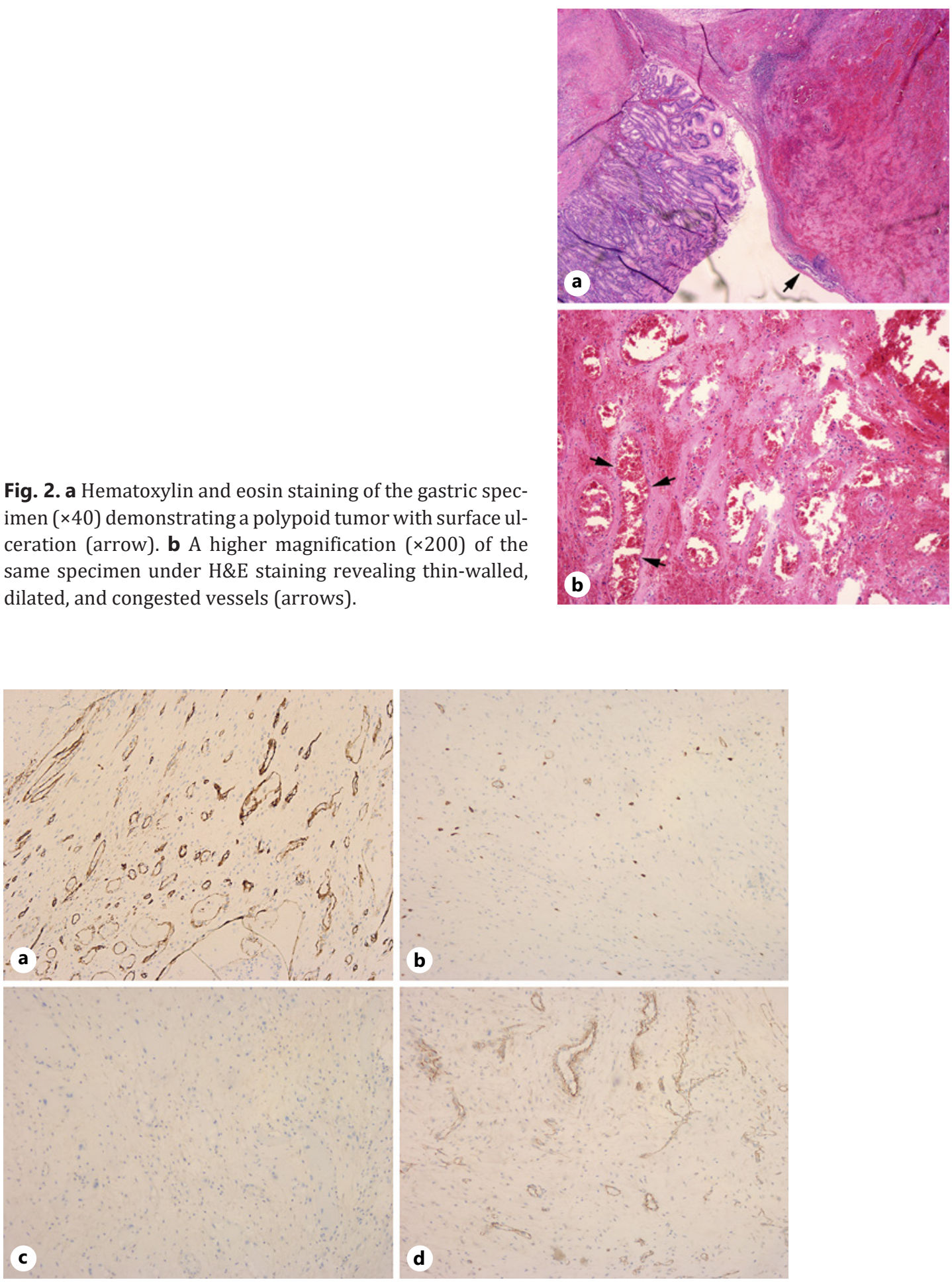

Fig. 3. Immunohistochemical study $(\times 100)$ showing positive staining for CD34 (a); immunohistochemical study $(\times 100)$ showing negative staining for CD117 (b); immunohistochemical study $(\times 100)$ showing negative staining for DOG-1 (c); immunohistochemical study $(\times 100)$ showing negative staining for betacatenin (d). 
syndrome and blue rubber bleb nevus syndrome [5, 7]. The cavernous subtype typically comprised larger diameter blood vessels with accompanying wider blood-filled spaces (caverns) as opposed to their capillary counterparts which consist of smaller blood vessels [1]. These properties make cavernous GHs more prone to rupture, resulting in significant bleeding. With respect to demographics, although there was no gender difference in prevalence [8], the lesion is predominantly reported in the pediatric [3, 9-11] and elderly [5, 8] populations which mostly do not overlap with those experiencing upper GI bleeding of other etiologies including peptic ulcers and varices.

Because GHs are often asymptomatic, most are identified incidentally due to advances in imaging modalities. Symptomatic GHs typically manifest as epigastric pain with associated GI bleeding, usually slow or occult bleeding from capillary-type tumors and heavy sudden onset, sometimes recurrent, bleeding from cavernous-type tumors which present with symptoms of anemia, hematemesis, and/or melena $[1,5,11]$. Severe hemorrhage due to rupture of cavernous GHs may lead to rapid hemodynamic decompensation and should be treated as a clinical emergency. The patient in our case also showed signs of hemodynamic compromise, which was treated with adequate fluid resuscitation and blood transfusion. It is vital to recognize the appearance of GH across multiple imaging modalities and differentiate $\mathrm{GH}$ from other gastric pathologies due to presentation of GH mimicking that of peptic ulcer disease. Other differential diagnoses comprise submucosal pathologies including leiomyomas, lipomas, GI stromal tumors, and varicose vessels. Endoscopic investigation is of utmost importance in patients presenting with upper GI bleeding. GHs typically appear as wellcircumscribed, vascular submucosal masses ranging from bluish-black to bright red in color with or without accompanying calcification $[2,6]$. This contrasts with the unusual endoscopic presentation in our case where the appearance of the vascular lesion mimicked that of a well demarcated II-b peptic ulcer. A diagnostic error made at this stage would have led to endoscopic removal of the blood clot-like mass via a cold snare technique as a routine treatment for PUD, thereby potentially causing rupture of the lesion or entrapment of the snare, both of which would have led to devastating consequences. Gastric ulcer is a relatively unlikely diagnosis among young females in their 20s [12]. Therefore, considering the appropriate treatment for a female patient without a history of PUD, the finding of a gastric ulcer raised a red flag regarding the possibility of another diagnosis. As a result, endoscopic intervention was fortunately avoided in our case.

Usage of imaging modalities such as abdominal CT and MRI is paramount in the evaluation of GHs as they demonstrate characteristic vascular enhancement patterns of hemangiomas $[4,6,11]$. Although there is existing literature advocating the presence of phleboliths on CT as being pathognomonic for GHs [1,3], this sign was absent in our case. Preoperative imaging studies also provide necessary insight into the extent of lesion and its relationship with surrounding anatomical structures. However, for GHs showing weak blood vessel signals, CT and MRI are unable to unequivocally differentiate GHs from other mesenchymal lesions. Alternatively, there is also literature delineating the efficacy of endoscopic ultrasound in assessing the extent of vascularity in cavernous GHs and other submucosal gastric tumors [13]. Nevertheless, this was not performed for our patient.

The presence of symptoms is the main indication for treatment in patients with GHs. Following supportive pharmacotherapy, surgical involvement is considered the mainstay curative treatment in adult patients with no reports of recurrence following complete resection [3,4]. Depending on the dimensions and location of the lesion, wedge resection and subtotal or total gastrectomy are all possible surgical options. Subtotal distal gastrectomy was performed in our case due to the antral location of the lesion. Endoscopic resection of GH is difficult due to its submucosal location and the probability of major hemorrhage because of the dense vascular nature of the lesion. However, therapeutic endoscopic intervention is 
not impossible for smaller lesions as 1 author has described a 14-mm cavernous GH being treated successfully via endoscopic resection [14]. Other nonsurgical treatments of GH including propranolol [15] and argon plasma coagulation [9] have also been described but only in infantile patients.

In conclusion, we report a case of isolated cavernous $\mathrm{GH}$ in an underreported age group with unusual endoscopic appearance mimicking that of PUD. While there is no gold standard, a combination of endoscopic evaluation and CT or MRI assessment provides valuable diagnostic insight, with surgery being curative. This case report not only highlights the rarity of such lesions and the possibility of atypical endoscopic presentation but also calls attention to the importance of considering GH as a potential differential diagnosis in young females with upper gastrointestinal bleeding for whom gastric ulcers are relatively rare.

\section{Statement of Ethics}

This study protocol was reviewed and approved by the Institutional Review Board of Shin Kong Wu Ho-Su Memorial Hospital, Approval No. 20210702R. Written informed consent was obtained from the patient for publication of the details of the medical case and any accompanying images.

\section{Conflict of Interest Statement}

The authors of this manuscript do not have any conflicts of interest to declare.

\section{Funding Sources}

This manuscript did not receive any funding.

\section{Author Contributions}

Andrew Sun - contribution in identification of case, acquisition of relevant data and drafting of the work, and final approval for publication. Jeffrey Sun - multiple revisions for intellectual content and final approval for publication. Cheuk-Kay Sun - acquisition, interpretation and analysis of data and images, and final approval for publication.

\section{Data Availability Statement}

All data generated or analyzed during this study are included in this article. Further enquiries can be directed to the corresponding author.

\section{References}

1 Zong L, Chen P, Shi GH, Wang L. Gastric cavernous hemangioma: a rare case with upper gastrointestinal bleeding. Oncol Lett. 2011;2(6):1073-5.

2 DiMarino AJ. Gastrointestinal disease: an endoscopic approach. Thorofare: SLACK Incorporated; 2002.

3 Abrahamson J, Shandling B. Intestinal hemangiomata in childhood and a syndrome for diagnosis: a collective review. J Pediatr Surg. 1973;8(4):487-95.

\section{Karger's}


4 Basbug M, Yavuz R, Dablan M, Baysal B, Gencoglu M, Yagmur Y. Isolated cavernous hemangioma: a rare benign lesion of the stomach. J Clin Med Res. 2012;4(5):354-7.

5 Bongiovi JJ Jr, Duffy JL. Gastric hemangioma associated with upper gastrointestinal bleeding. Arch Surg. 1967; 95(1):93-8.

6 Levy AD, Abbott RM, Rohrmann CA Jr, Frazier AA, Kende A. Gastrointestinal hemangiomas: imaging findings with pathologic correlation in pediatric and adult patients. AJR Am J Roentgenol. 2001;177(5):1073-81.

7 Yamaguchi K, Kato Y, Maeda S, Kitamura K. Cavernous hemangioma of the stomach: a case report and review of the literature. Gastroenterol Jpn. 1990;25(4):489-93.

8 Handra-Luca A, Montgomery E. Vascular malformations and hemangiolymphangiomas of the gastrointestinal tract: morphological features and clinical impact. Int J Clin Exp Pathol. 2011;4(5):430-43.

9 Lee YA, Chun P, Hwang EH, Lee YJ, Kim CW, Park JH. Gastric hemangioma treated with argon plasma coagulation in a newborn infant. Pediatr Gastroenterol Hepatol Nutr. 2017;20(2):134-7.

10 Menon P, Rao KL, Bhasin S, Vanitha V, Thapa BR, Lal A, et al. Giant isolated cavernous hemangioma of the stomach. J Pediatr Surg. 2007;42(4):747-9.

11 Attash SM, Ali MS, Al-Nuaimy HA. Isolated cavernous haemangioma of the stomach in a 3-year-old child: an unusual cause of upper GI bleeding. BMJ Case Rep. 2012;2012.

12 Aro P, Storskrubb T, Ronkainen J, Bolling-Sternevald E, Engstrand L, Vieth M, et al. Peptic ulcer disease in a general adult population: the Kalixanda study: a random population-based study. Am J Epidemiol. 2006; 163(11):1025-34.

$13 \mathrm{Hu}$ J, Sao H, Sun S. Role of endoscopic ultrasound for the diagnosis of isolated gastric cavernous haemangioma. J Clin Diagn Res. 2016;10(9):XD03-4.

14 Arafa UA, Fujiwara Y, Shiba M, Higuchi K, Wakasa K, Arakawa T. Endoscopic resection of a cavernous haemangioma of the stomach. Dig Liver Dis. 2002;34(11):808-11.

15 Kaya H, Gokce IK, Gungor S, Turgut H, Ozdemir R. A newborn with gastric hemangioma treated using propranolol. Pediatr Gastroenterol Hepatol Nutr. 2018;21(4):341-6. 